\title{
Jupyter in Computational Science
}

\author{
Hans Fangohr ${ }^{0}$, Max Planck Institute for the Structure and Dynamics of Matter, Hamburg, 22761, Germany \\ Thomas Kluyver D, European XFEL, Schenefeld, 22869, Germany \\ Massimo DiPierro, DePaul University, Chicago, IL, 60604, USA
}

\section{N} otebook interfaces-documents combining executable code with output and notes-first became popular as part of computational mathematics software such as Mathematica and Maple. The Jupyter Notebook, which began as part of the IPython project in 2012, is an open source notebook that can be used with a wide range of generalpurpose programming languages.

Before notebooks, a scientist working with Python code, for instance, might have used a mixture of script files and code typed into an interactive shell. The shell is good for rapid experimentation, but the code and results are typically transient, and a linear record of everything that was tried would be long and not very clear. The notebook interface combines the convenience of the shell with some of the benefits of saving and editing code in a file, while also incorporating results, including rich output, such as plots, in a document that can be shared with others.

The Jupyter Notebook is used through a web browser. Although it is often run locally, on a desktop or a laptop, this design means that it can also be used remotely, so the computation occurs, and the notebook files are saved, on an institutional server, a high-performance computing facility or in the cloud. This simplifies access to data and computational power, while also allowing researchers to work without installing any special software on their own computer: specialized research software environments can be provided on the server, and the researcher can access those with a standard web browser from their computer.

These advantages have led to the rapid uptake of Jupyter Notebooks in many kinds of research. The articles in this Special Issue highlight this breadth, with the authors representing various scientific fields. But more importantly, they describe different aspects

1521-9615 ( 2021 IEEE

Digital Object Identifier 10.1109/MCSE.2021.3059494

Date of current version 25 March 2021. of using notebooks in practice, in ways that are applicable beyond a single field.

We open this Special Issue with an invited article by Brian Granger and Fernando Perez-two of the cofounders and leaders of Project Jupyter. Starting from the origins of the project, they introduce the main ideas behind Jupyter Notebooks, and explore the question of why Jupyter Notebooks have been so useful to such a wide range of users. They have three key messages. The first is that notebooks are centered around the humans using them and building knowledge with them. Next, notebooks provide a write-eval-think loop that lets the user have a conversation with the computer and the system under study, which can be turned into a persistent narrative of computational exploration. The third idea is that Project Jupyter is more than software: it is a community that is nourished deliberately by its members and leaders.

The following five articles in this Special Issue illustrate the key features of Project Jupyter effectively. They show us a small sample of where researchers can go when empowered by the tool, and represent a range of scientific domains.

Juneau et al. describe how Jupyter has been used to "bring the compute to the data" in astrophysics, allowing geographically distributed teams to work efficiently on large datasets. Their platform is also used for education and training, including giving school students a realistic taste of modern science.

Abernathey et al., of the Pangeo project, present a similar scenario with a focus on data from the geosciences. They have enabled analysis of big datasets on public cloud platforms, facilitating a more widely accessible "pay as you go" style of analysis without the high fixed costs of buying and setting up powerful computing and storage hardware. Their discussion of best practices includes details of the different data formats required for efficient access to data in cloud object stores rather than local file systems.

Beg et al. describe features of Jupyter Notebooks and Project Jupyter that help scientists make their research reproducible. In particular, the work focuses on the use of computer simulation and mathematical 
experiments for research. The self-documenting qualities of the notebook - where the response to a code cell can be archived in the notebook-are an important aspect. The article addresses wider questions, including the use of legacy computational tools, exploitation of HPC resources, and creation of executable notebooks to accompany publications.

Mooers describes the use of a snippet library in the context of molecular structure visualization. Using a Python interface, the PyMOL visualization application can be driven through commands to visualize molecular structures such as proteins and nucleic acids. By using those commands from the Jupyter Notebook, a reproducible record of analysis and visualizations can be created. The article focuses on making this process more user friendly and efficient by developing a snippet library, which provides a wide selection of precomposed and commonly used PyMOL commands, as a JupyterLab extension. These commands can be selected via hierarchical pull-down menus rather than having to be typed from memory. The article discusses the benefits of this approach more generally.

Watters describes a widget that can display 3-D objects using webGL, while the back-end processes the scene using a data visualization pipeline. In this case, the front-end takes advantage of the client GPU for visualization of the widget, while the back-end takes advantage of whatever computing resources are accessible to Python.

The articles for this Special Issue were all invited submissions, in most cases from selected presentations given at JupyterCon in October 2020. Each article was reviewed by three independent reviewers. The guest editors are grateful to Ryan Abernathey, Luca de Alfaro, Hannah Bruce MacDonald, Christopher Cave-Ayland, Mike Croucher, Marco Della Vedova, Michael Donahue, Vidar Fauske, Jeremy Frey, Konrad Hinsen, Alistair Miles, Arik Mitschang, Blaine Mooers, Samual Munday, Chelsea Parlett,
Prabhu Ramachandran, John Readey, Petr Škoda, and James Tocknell for their work as reviewers, along with other reviewers who preferred not to be named. The article by Brian Granger and Fernando Perez was invited by the Editor-in-Chief, and reviewed by the editors of this Special Issue.

HANS FANGOHR is currently the head of the Computational Science Group, Max Planck Institute for the Structure and Dynamics of Matter, Hamburg, Germany, and is also a professor of computational modeling with the University of Southampton, U.K. He authored more than 150 scientific articles in computational science and materials modeling, several open-source software projects, and a text book Python for Computational Science and Engineering. He received the Ph.D. degree in computer science in 2002. Contact him at hans.fangohr@mpsd.mpg.de.

THOMAS KLUYVER is currently a software engineer with European XFEL. His research focuses on various parts of the open source and scientific computing ecosystems, including the Jupyter \& IPython projects. He received the Ph.D. degree in plant sciences from the University of Sheffield in 2013. Contact him at thomas.kluyver@xfel.eu.

MASSIMO DI PIERRO is currently a professor of computer science with DePaul University. He is also the lead developer of many open-source projects including web2py, py4web, and pydal. He has authored more than 70 articles in physics, computer science, and finance and has authored or coauthored three books. His research interests include numerical algorithms, high-performance computing, and machine learning. He received the Ph.D. degree in theoretical physics from the University of Southampton. Contact him at massimo.dipierro@gmail.com. 